\title{
On the Performance of a Medium Access Scheme Based on EY-NPMA in a High Bitrate Wireless LAN
}

\author{
G. Dimitriadis and F.-N. Pavlidou \\ Department of Electrical and Computer Engineering \\ Aristotle University of Thessaloniki \\ 54124 Thessaloniki, Greece \\ \{gedimitr, niovi\}@eng. auth.gr
}

\begin{abstract}
Wireless LANs provide an efficient and inexpensive way for the creation of networks that do not constrain the users' mobility. Furthermore, advanced physical layer techniques have allowed wireless LANs to support bitrates that until recently were attainable only in wired networks. The growing penetration of WLANs in the market, as well as the wider spectrum of applications that these kinds of networks are called to support, demands the introduction of new methods for efficient medium access. In this paper, we propose and evaluate the performance of a medium access scheme that is based on the EY-NPMA protocol for medium access, showing through simulation the good characteristics of the proposed scheme.
\end{abstract}

\section{Introduction}

Wireless LANs provide an efficient and inexpensive way for the creation of networks that do not constrain the users' mobility. Spontaneous, hassle free communication has become possible through ad hoc networks, while infrastructure based wireless LANs have permitted true flexibility and freedom without demanding installation of new cables or retrofitting. Furthermore, advanced physical layer techniques have allowed wireless LANs to support bitrates that until recently were attainable only in their wired counterparts. The growing penetration of WLANs in the market, as well as the wider spectrum of applications that these kinds of networks are called to support, demands the introduction of new methods for efficient medium access, since it is the MAC who defines how efficiently the available raw bandwidth is used. In this paper we present and evaluate the performance of of a modified version of the EY-NPMA medium access scheme, a protocol that is part of the HIPERLAN [1] wireless networking standard. The rest of the paper is structured as follows. In section 2 we provide a brief presentation of the background on medium access for wireless LANs. In section 3, we present the proposed medium access scheme, while sketching the mechanics of the base EY-NPMA protocol. In section 4, the simulation results are presented and finally, section 5 concludes the paper. 


\section{Background Work}

A medium access scheme must possess three characteristics in order to be an efficient one. It must ensure that the common medium is fairly shared between the nodes that consist the network, provide explicit quality of service (QoS) guarantees for different classes of traffic and finally maximize the medium utilization by minimizing the wasted capacity that is caused by packet collisions and/or overhead. Furthermore, all of the above should be implemented in a design that is both robust and simple. In the field of wireless LANs, there are two dominating families of protocols for medium access. The first one includes those that are based on contention, while the other includes the protocols that are based on the paradigm of dynamic assignment.

Contention based medium access schemes represent a paradigm that has been traditionally connected with Local Area Networks (mostly because of the widespread deployment of IEEE 802.3 - Ethernet). According to protocols belonging to this family, packet transmissions take place in a completely stochastic way, with minimal or totally absent coordination between the nodes participating in the network. Consequently, there exists a probability that multiple transmissions take place simultaneously, resulting in erroneous reception. To reduce such occurences, the concept of carrier sensing is widely employed, since it prevents network nodes from transmitting, when they sense that a transmission is already in progress. For technical reasons, the variant of carrier sensing that uses collision detection (CSMA/CD) cannot be applied in wireless networks, so in order to minimize the impact of collisions, the technique of collision avoidance (CSMA/CA) is widely used, initially proposed by Karn in [2]. According to this scheme, packet collisions are restricted only between small Request-To-Send, Clear-To-Send packets (RTS/CTS), while the actual data packets are transmitted collision-free. Furthermore, this handshake between transmitter and receiver effectively mitigates the hidden terminal problem [3].

Contention based medium access schemes are particularly well fit for bursty traffic (which is the case for most data communications), but generally they are not well suited for providing quality of service guarantees for different traffic classes. Regarding throughput, as a rule contention based protocols perform best under light to medium traffic loads, since the rate of collisions increases sharply as the traffic load exceeds a threshold. However, in the last few years, the importance of service differentiation has triggered intense research activity on embedding quality of service (QoS) capabilities in contention based medium access schemes. Protocols that are QoS aware include Blackburst proposed by Sobrinho and Krishnakumar in [6], DFS (Distributed Fair Scheduling) [7], the under standardization by the 802.11e task group medium access protocol EDCF (Enhanced Distributed Coordinator Function) [5], as well as the aforementioned EY-NPMA protocol. On the other hand, DCF (Distributed Coordinator Fuction) of the original IEEE 802.11 [4] standard for wireless LANs is insensitive to different traffic classes.

Medium access schemes following the dynamic assignment approach gain access to the common medium either via an arbitrator (e.g. a terminal that 


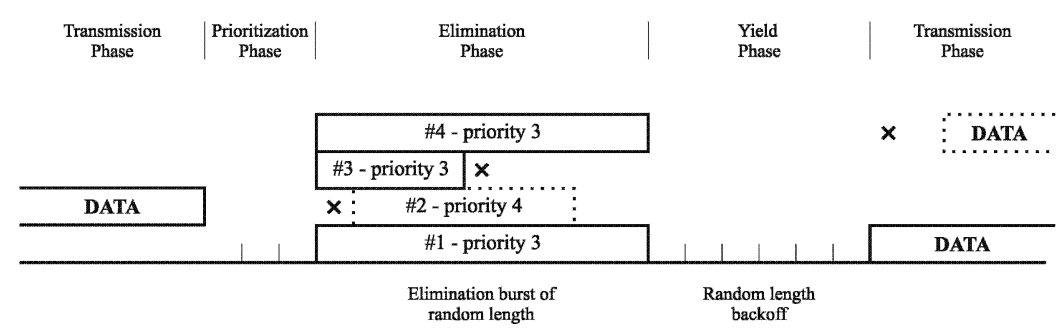

Fig. 1. EY-NPMA's synchronized channel access cycle

polls each station in a round-robin fashion) or through a well-defined process that ensures that only one terminal at a time has permission to gain access to the channel (e.g. reservation or token passing schemes). An important difference from contention based medium access protocols is that with dynamic assignment schemes there is usually a need for a management entity, undertaking the role of polling, creating tokens when they get lost and validating reservations. In infrastructure based wireless LANs, the access point (AP) is the obvious candidate for such a role, but in ad hoc networks a node is dynamically chosen to become channel coordinator, usually through clustering techniques. Compared to contention based medium access schemes, the performance of dynamic assignment protocols does not deteriorate when the traffic load increases, while the deterministic access to the channel inherently provides quality of service support. On the other hand, the fact that each station must explicitly acquire access permission (i.e. get polled or capture the token), introduces a latency that is mostly notable in cases of light traffic, while traffic load asymmetries tend to deteriorate the performance of these protocols. Also, the hidden terminal problem proves to be more difficult to alleviate in dynamic assignment protocols, rather than in contention based ones. Protocols that follow the dynamic assignment paradigm are the medium access layers of the HIPERLAN/2 [8] and Bluetooth [9] standard, as well as the PCF (Point Coordinator Function) access mode of the IEEE 802.11 standard.

\section{Protocol Model}

EY-NPMA stands for Elimination-Yield Non-pre-emptive Priority Multiple Access. It is a contention based protocol that has been standardised under ETSI's HIPERLAN standard for wireless LANs. EY-NPMA provides excellent support for different classes of traffic regarding quality of service and demonstrates very low collision rates. In the following subsections, we will present the inner workings of the base EY-NPMA scheme and after that the proposed modified scheme.

\subsection{EY-NPMA}

According to EY-NPMA, the channel access cycle consists of four distinct phases: Prioritization, Elimination, Yield and Data Transmission. In prioritization, EY- 
NPMA recognizes five distinct priorities from 0 to 4 , with 0 being the highest priority. The cycle begins with each station having data to transmit sensing the channel for as many slots as the priority of the packet in its buffer. All stations that succesully sense the channel as idle for the whole interval proceed to the next phase, the elimination phase. Those that do not, exit the contention process and wait for the next synchronized channel condition to make another attempt. During the elimination phase, each station transmits an energy burst of random length. These bursts ensure that only the stations having the highest priority data at a time proceed to the elimination phase. As soon as a station finishes bursting, it immediately senses the channel. If the channel is sensed as idle, the station proceeds to the next phase. Otherwise, it leaves the cycle. During the yield phase, the stations that survived the two previous ones, back off for a random number of slots. The station that backs off for the shortest interval eventually gets access of the channel for data transmission. All other stations sense the beginning of the transmission and refrain from transmitting. Each phase reduces the number of stations that remain into the contention process, so that (hopefully) the station that will commence transmitting data in a given time will be unique. In figure 1, we present a typical synchronized channel access cycle. Solid line boxes represent actual transmissions, while dashed line boxes represent projected transmissions that did not take place because the station left the contention process. The $\mathrm{X}$ marks show when and why a station left the cycle.

Through this four-phases cycle, EY-NPMA manages to provide a low and quasi-constant rate of collisions. The parametres chosen in the HIPERLAN standard (maximum number of slots for bursting and backing off, etc) aimed at a target collision rate of $3.5 \%$ for a population of 256 simultaneously contending nodes, a figure that guaranteed that for most practical situations communications would be collision-free. However, this important merit of EY-NPMA turns out to also be its most severe disadvantage. In order to achieve such a low rate of collisions, a large number of slots is allocated to the elimination and yield phase and thus are being experienced as overhead. As advances in the physical layer lead to higher bitrates, this effect becomes even more intense. Typical values for the duration of each slot are around $10 \mu \mathrm{s}$, a value which at high bitrates becomes a significant fraction of the time needed to transmit the actual data payload. Because of the wireless environment, but also for technical reasons, there is a lower limit to the slot duration. Propagation delay and delay spread demand that the slot duration for both elimination and yielding exceeds a certain threshold, while especially for bursting, rise and fall times of each burst place this threshold even higher. In the following subsection, we describe a medium access scheme based on the original EY-NPMA protocol, that manages to achieve a more efficient utilization of the shared medium.

\subsection{Proposed Scheme}

The proposed medium access scheme features a different structure for the prioritization phase and a mechanism for dynamically promoting the packet priority 


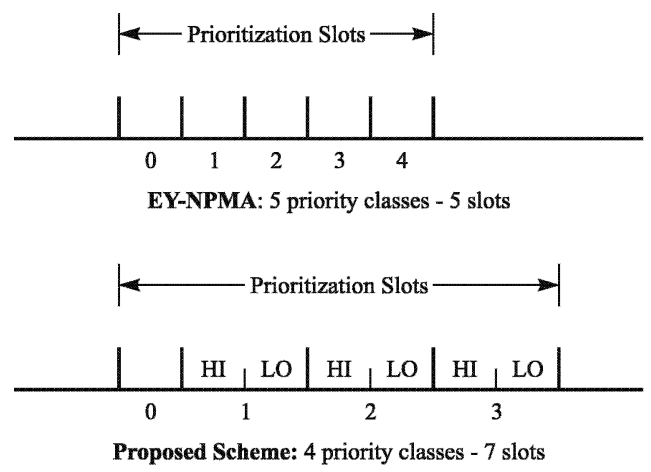

Fig. 2. Structure of the prioritization phase for both schemes

for packets that have survived the elimination process, but did not survive the yield phase. Instead of 5 priority classes, we propose a scheme with 4 priorities. The three lower ones consist of two subclasses, namely a low and a high. The structure of the prioritization slots for the two schemes is depicted in figure 2 . A data packet of priority $x$ that is placed in the transmission buffer of the MAC contoller by an upper layer is automatically labeled as being $x$-low priority. Let $x$-low be the highest priority when a channel access condition occurs and a number of $N$ stations enter the contention process with all $N$ stations commencing bursting at the same slot. At the end of the elimination phase, a fraction of the initial population, $N_{s}$, will have survived elimination and will choose a random number of slots to backoff. At this point all $N_{s}$ stations switch their packets priorities from $x$-low to $x$-high. At the next channel access cycle, through the prioritization phase only the $N_{s}$ stations will enter the elimination phase, in contrast to the at least $N-1$ stations that would enter contention according to the base EY-NPMA scheme. Access cycles at $x$-low priority will be postponed, until there are no more $x$-high packets, which will happen at the end of at least $N_{s}$ cycles. When there are no more packets of $x$-high priority, an access cycle of $x$-low will follow, and the whole process will be repeated.

Furthermore, since at $x$-high priority level there are much fewer contending nodes, a reasonable rate of collisions can be achieved with fewer slots dedicated to elimination and yielding, than in the case of $x$-low priority. Hence, by demanding the all $x$-high cycles employ fewer cycles for the two contention resolution phases, we are led to better medium utilization figures, since for the same data payload the access cycles become shorter. The cost of this modification is the reduction of the traffic classes by one and the addition of two extra slots for the prioritization phase. Consequently, when a big population of stations wants to send data in the same base priority class $x$, according to the base EY-NPMA scheme in each access cycle this big population will content for channel access. On the contrary, with our modification the whole population contents for one cycle, while for a number of subsequent cycles only a subset of these stations will participate in the contention process. Furthermore, the protocol's behaviour to the base priority 


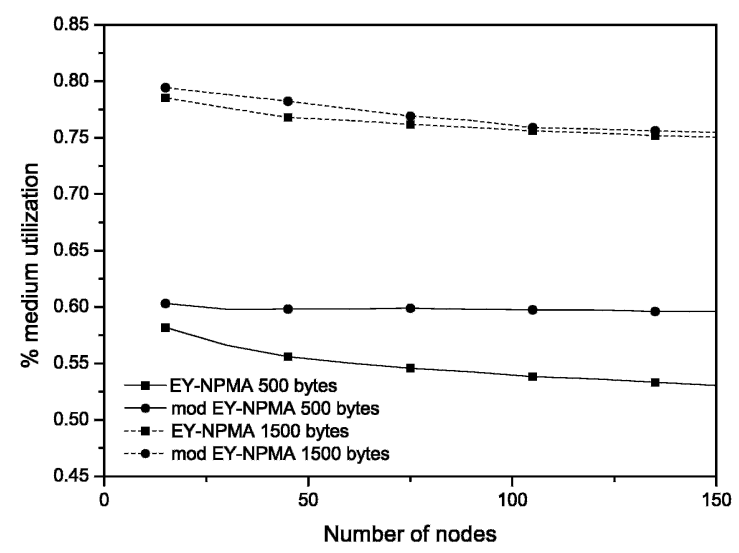

Fig. 3. Medium utilization vs number of contending nodes

classes does not change by our modification. That is a priority 3 packet will always be of lower priority than a priority 2 packet, no matter what subclass low or high - the packets happen to be.

\section{Simulation}

\subsection{Simulation Scenario}

The simulation trials for both the base and the modified EY-NPMA scheme were conducted using a custom simulator developed by the authors in $\mathrm{C}++$. For the needs of this work, two scenarios were examined and evaluated. The first one considered the performance of a high bitrate wireless LAN as the number of contending nodes increased, while the second dealt with the transport capabilities of the same wireless LAN for two different traffic classes as the offered load increased. For both scenarios we assumed a fully connected topology, that is a network where between each pair of nodes there exists a bidirectional link. Consequently, with the above assumption we ruled out the existence of any hidden nodes and also avoided routing of data packets as would be the case in a multihop wireless network. The channel capacity was set to $20 \mathrm{Mbps}$, while in the case of the base EY-NPMA protocol the maximum number of elimination slots (bursting) was set to 9 and the maximum number of slots for the yielding phase (backoff) was set to 6 . For the proposed scheme, 5 slots were allocated to elimination and 6 slots to yielding for $x$-low priority packets, while for $x$-high priority cycles, 3 slots were allocated for elimination and 4 slots for yielding. The duration of a slot for both phases was set to $10 \mu \mathrm{s}$.

For the first set of experiments, an increasing number of nodes contend for channel access. A high rate packet generator of priority 1 is attached to each node, so that their transmission buffer is never empty. For this scenario, we 


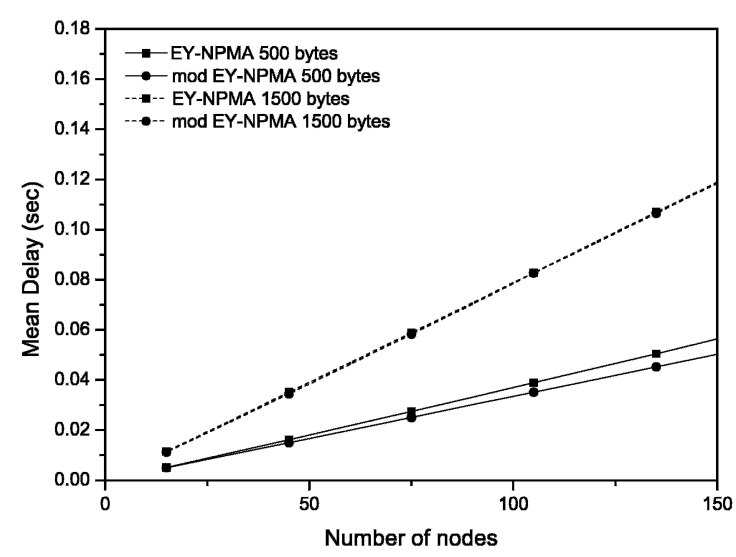

Fig. 4. Mean packet delay vs number of contending nodes

experimented with two packet sizes, the first being 500 bytes and the second 1500 bytes. The network performance was evaluated using two metrics, namely medium utilization and mean packet delay. Medium utilization is defined as the percentage of time that is used for the successful transmission of actual data frames, while the metric of mean delay expresses the time needed between placement of a data packet in the transmission buffer and positive acknowledgement of its reception. In the second scenario, we examine the performance of a network consisting of 100 nodes, under a variable offered load. The network population is divided into two groups: a group of 40 nodes generating high priority data packets (priority 1), and a group of 60 nodes generating low priority data packets (priority 2). For both priority classes, the packet size is equal to 500 bytes, while the packet generation rate for high priority traffic is 5 time the correspondent rate for low priority traffic. Consequently, for any given value of aggregate offered load, $23 \%$ is due to low priority sources and $77 \%$ is due to high priority sources. For this scenario, the network performance was measured also in terms of medium utilization and mean packet delay.

\subsection{Simulation Results}

In figures 3 and 4 , we present the results of the first scenario. Figure 3 depicts the medium utilization for a variable number of simltaneously contending nodes. The base EY-NPMA scheme shows a poorer performance than the modified medium access scheme for both 500 and 1500 bytes of packet size. Furthermore, the difference in performance increases as data packets become shorter. In the case of 1500 bytes long data packets, the modified EY-NPMA scheme shows an increase in medium utilization of $2.4 \%$, while for 500 bytes long packets, the correspondent increase is somewhat less than $10 \%$. This phenomenon is accounted to the importance of the slot duration relative to the time needed for a data frame transmission. The proposed scheme employs fewer slots for the 


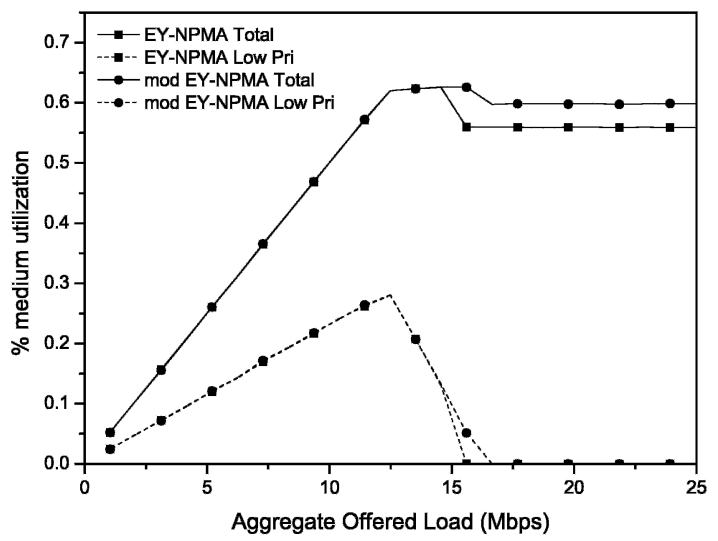

Fig. 5. Medium utilization vs aggregate offered load

contention resolution process than the base EY-NPMA protocol. The impact of these savings is more evident in the case of short data packets, where the slot duration is a more significant fraction of the time needed for the transmission of the actual data packet, than in the case of long data packets.

In this figure, three out of four curves show a decreasing trend. As was expected, in the case of the base EY-NPMA scheme, our simulations showed that the collisions rate remained quasi-constant while the node population increased. The decrease in medium utilization is owed to the fact that when the number of contending nodes increases, the average length of the elimination phase increases too, since each node randomly chooses a number of slots for bursting according to a geometric distribution. This increase of the elimination phase is translated to increases in overhead and hence to worse medium utilization. In the case of the modified EY-NPMA scheme, the number of nodes that survive the elimination phase is larger, since we allocated fewer slots for bursting. This had two conflicting results: In $x$-low there was a higher collisions probability, but more data frames were transmitted in $x$-high, achieving data transmissions with favorable collisions probability in shorter cycles. In the case of short data packets, these two phenomena reach an equilibrium and hence the medium utilization is almost independent of the number of contending nodes. In the case of long data frames, however, the effect of the increased collisions rate is slightly more intense and a decrease in medium utilization is experienced.

In figure 4, we present the mean delay experienced by a packet between acceptance by the MAC layer and positive reception acknowledgement. For both packet sizes, the modified EY-NPMA scheme showed an increase in performance compared to the base medium access protocol. For the same reasons that were reported in the previous paragraphs, there are more significant differences in performance in the case of short packets, rather in the case of packets that are 1500 bytes long. The increase in mean packet delay as the population size 


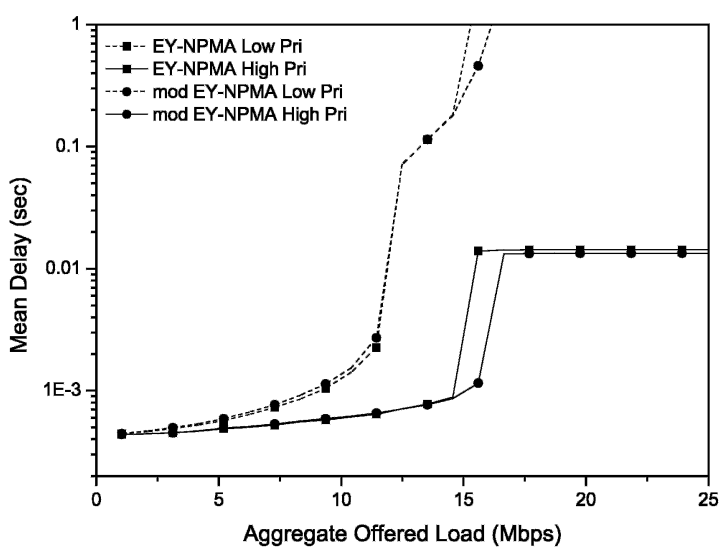

Fig. 6. Mean packet delay vs aggregate offered load

increases shows a linear behaviour for both schemes. This is primarily attributed to the fact that the same capacity is shared by a larger number of nodes and not to an increase in collisions, something that is also confirmed by the results of figure 3.

Figures 5 and 6 depict the results gained from the second scenario simulations. In figure 5 the medium utilization versus variable aggregate load is presented. For each scheme the curves of total medium utilization and medium utilization due to low priority traffic are drawn on the graph. The medium utilization by the high priority traffic can easily be deducted by substracting the two curves. In this figure the medium utilization for both schemes initially increases linearly as the offered load increases. However, as hinted by figure 3 , in the case of the base EY-NPMA scheme the network reaches its saturation point earlier than in the case of the modified scheme, while in steady state EY-NPMA also shows a worse performance. Beyond the saturation point, low priority traffic is gradually phased out, until eventually it is completely starved. However, in the case of the modified EY-NPMA scheme, starvation of low priority traffic occurs later, than in the case of the base protocol.

In figure 6, we present the mean packet delay for the same scenario. We see that for both schemes the curves of low and high priority data follow a similar trend. When the network operates beneath the saturation point, packets experience a mean delay that is less than $5 \mathrm{~ms}$. We also notice that beneath the saturation point, the mean delay of the proposed scheme is slightly higher than in the case of the base EY-NPMA scheme, something that is especially evident in the case of low priority traffic. This is owed to the fact that even though both classes have the same priority (1 and 2), in the case of the modified EY-NPMA protocol nodes commence bursting 1 or 2 slots later, for high and low priority traffic respectively, than in the case of the base EY-NPMA scheme. Since there is no intense contention at this point of operation, almost all cycles 
are at $x$-low and hence the access cycles are longer in the case of the modified scheme. However, as the network approaches and passes the saturation point the modified scheme provides better delay figures, since an increasing number of shorter cycles are done in $x$-high priority.

\section{Conclusions}

In this paper we presented and evaluated the perfomance of EY-NPMA in a high bitrate wireless LAN and also proposed a modification of the base scheme that manages to achieve good performance both in terms of medium utilization and mean packet delay. The guaranteed low collisions rate of EY-NPMA comes at a cost that becomes higher, as the network speed increases. The proposed scheme reaches a balance between overhead and rate of collisions resulting in better utilization of the available capacity. Simulation trials for two different scenarios confirm the positive impact of these modifications. However, these first results also show that the proposed scheme has still room for improvement. Our future work will primarily concentrate on optimizing the proposed scheme and also compare it to other QoS aware medium access protocols.

\section{References}

[1] European Telecommunications Standards Institute (ETSI), ETSI EN 300652 V1.2.1: Broadband Radio Access Networks (BRAN): HIgh PERformance Local Area Network (HIPERLAN) Type I: Functional Specification, 1998

[2] P. Karn, "MACA - A New Channel Access Protocol for Packet Radio," ARRL/CRRL Amateur Radio Ninth Conputer Networking Conf., pp. 134-140, 1990

[3] F. Tobagi, L. Kleinrock, "Packet Switching in Radio Channels: Part II - the Hidden Terminal Problem in Carrier Sense Multiple-Access and the Busy-Tone Solution," IEEE Transactions in Communications, vol. 23, no. 12, pp. 1417-1433, 1975

[4] The Institute for Electrical and Electronics Engineers (IEEE), IEEE Std 802.11 - Wireless LAN Medium Access Control (MAC) and Physical Layer (PHY) specifications, 1999

[5] M. Benveniste et al., EDCF Proposed Draft Text. IEEE working document 802.1101/131r1, 2001

[6] J. Sobrinho, A. Krishnakumar, "Quality-of-Service in Ad Hoc Carrier Sense Multiple Access Networks," IEEE Journal on Selected Areas in Communications, vol. 17 , no. 8, pp. $1353-1368,1999$

[7] N. Vaidya et al., "Distributed Fair Scheduling in a Wireless LAN," Sixth Annual International Conference on Mobile Computing and Networking, USA, 2000

[8] European Telecommunications Standards Institute (ETSI), DTS/BRAN0020004-1 V0.m: Broadband Radio Access Networks (BRAN): HIgh PERformance Local Area Network (HIPERLAN) Type II: Data Link Control (DLC) Layer: Part 1: Basic Transport Functions, 1999

[9] Bluetooth Special Interest Group, Specification of the Bluetooth System - Core, V1.0B, 1999 